\title{
Morphological Characterization of Biocontrol Isolates of Trichoderma to Study the Correlation between Morphological Characters and Biocontrol Efficacy
}

\author{
Muthu Kumar. $A^{1, a}$ and Pratibha Sharma ${ }^{2}$ \\ ${ }^{1}$ Forest Wood Protection Division, Institute of Wood Science \& Technology, \\ Bangalore, India - 560003 \\ ${ }^{2}$ Division of Plant Pathology, Indian Agricultural Research Institute, \\ Pusa, New Delhi, India- 1100012 \\ atouchurpearl@gmail.com
}

Keywords: Morphological characterization, Trichoderma, taxonomy, biocontrol, antagonism.

\begin{abstract}
The morphological characterization was carried out for 5 isolates of Trichoderma harzianum and 7 isolates of Trichoderma viride and tested for their biocontrol efficacy. The isolates belonging to T.harzianum were analogous in colony colour, culture smell, mycelial colour, conidiation, conidial shape, conidial wall and conidial colour. Correspondingly the isolates of T.viride showed certain similarity in colony colour, colony edge, culture smell, conidiophore branching, conidial wall, conidial colour and chlamydospores. Inter specific differences through cluster analysis based on morphological characters grouped the twelve isolates into three major clusters where all the isolates of T.harzianum formed a single cluster while the isolates of T.viride were bifurcated into two groups. The clustering was substantiated by similarity index which showed maximum similarity among T.harzianum isolates with only less than $20 \%$ variation among themselves. Similarly the clusters having isolates of T.viride also had less variation within them. The biocontrol efficacy of these twelve isolates of Trichoderma was experimented by dual culture test under laboratory condition and there existed some relation between the biocontrol efficacy of these isolates and morphology.
\end{abstract}

\section{Introduction}

Identification based on morphological characters consent a relatively simple method for classification of Trichoderma as genus, but the species perceptions are complex to construe and there is considerable confusion over the application of specific names. Bisby could identify only one species T.viride after examining several isolates and collection of Trichoderma without finding any reliable characters to distinguish them, he concluded Trichoderma as a monotypic genus. It was only few years ago the factual character of Trichoderma has been recognized [1]. Pioneers in Trichoderma like Rifai and Bissett observed certain cultural characters that could be used for identification and description of these species viz, tuft or cushion of hyphae on natural substrate composed of conidiophores, spores and some sterile hyphae, conidiophores indefinite, branched or unbranched hyphae bearing phialides laterally or terminally, phialides oust by heads rarely short or in chains, spore hyaline or brightly colored, one celled. Nonetheless, class affinities of the genus for the development of species are still very slow [14, 3, 4, and 5]. Rifai classified the Trichoderma into nine species aggregates [14], further it was elaborated by Bissett [2, 3, 4 and 5], covering thirty five species, their classification reflected the importance of microscopic characters for delimiting the Trichoderma species.

Members of the fungal genus Trichoderma were found to be useful as effective biological control agents for many diseases caused by soil borne pathogens. Weindling gave the first report on Trichoderma as a potential biocontrol agent [22], since then various workers have speculated the existence of biological control ability of Trichoderma for over seventy years [9]. Trichoderma species can act as biocontrol agents through different synergistic mechanisms. However, it is difficult to predict the degree of synergism and the behavior of a biocontrol agent in a natural 
pathosystem. Considering that environmental conditions are important, the right selection of biocontrol agents, which begins with a safe characterization of biocontrol strains in the new taxonomic schemes of Trichoderma, is equally important since the exact identification of strains to the species level is the first step in utilizing the full potential of fungi in specific applications [11]. However the taxonomic status of this species is imprecise and the criteria used to classify and identify strains so far do not provide sufficient discrimination, especially with those isolates of interest in biocontrol programmes. Therefore the present study enumerated to characterize the cryptic species of Trichoderma taxa associated with biological control of certain soil borne pathogens based on morphological characters.

\section{Material and methods}

\section{Morphological characterization}

The five isolates of T.harzianum and seven isolates of T.viride examined for this study are listed in Table 1. All the isolates taken for study were already classified as Trichoderma harzianum and Trichoderma viride through biochemical analysis based on their toxicity over the plant pathogens and was reported as potential biocontrol agents [16].

Table 1. List of the Trichoderma isolates taken for the study

\begin{tabular}{|c|c|c|}
\hline No. & Isolate name & Species \\
\hline 1 & Th3 & T.harzianum \\
\hline 2 & Th10 & T.harzianum \\
\hline 3 & Th30 & T.harzianum \\
\hline 4 & Th31 & T.harzianum \\
\hline 5 & ThAg & T.harzianum \\
\hline 6 & Tv2 & T.viride \\
\hline 7 & Tv4 & T.viride \\
\hline 8 & Tv12 & T.viride \\
\hline 9 & Tv15 & T.viride \\
\hline 10 & Tv32 & T.viride \\
\hline 11 & TvChen & T.viride \\
\hline 12 & TvNir & T.viride \\
\hline
\end{tabular}

PDA with low sugar medium was used for the manipulation of growth rate of different isolates [13]. Few days later after the colonies become visible, mycelial mat of about $5 \mathrm{~mm}$ diam was taken from the actively growing edge of the colony and inoculated onto freshly prepared medium. The transfer of the mycelial mat should be before the culture start producing conidia. The mat was placed approximately $1.5 \mathrm{~cm}$ from the edge of the petriplate and the plates were incubated under darkness at $25^{\circ} \mathrm{C}$. They were examined at 25 hourly intervals and observed for growth rate (colony radius from the edge of mat), colony edge, colour, smell and reverse colony colour. The growth tests were repeated four times at roughly weekly intervals and the average readings were taken. All micro morphological data were taken within one week from colonies grown on PDA containing the antibiotics streptomycin at $25^{\circ} \mathrm{C}$.

Every measurement of micro morphological characters were taken from material that was immersed in drop of $3 \%$ aq. $\mathrm{KOH}$, which was consequently substituted by water since the microscopic preparation desiccated. For direct microscopic observations, 20 units of every character 
were measured with the exclusion of chlamydospores. Light and phase contrast microscope were employed in the present study. The morphological characterization was studied by the observations made from the microscopic slides, with verification of the key provided by Rifai and Bissett [14, 2, 3,4 , and 5]. Other cultural characters like colony color, growth rate, colony edge and culture smell were also studied.

\section{Statistical analysis}

The observed phenetic characters were used as descriptors and the variation present within each descriptor were called as descriptor states. For our convenience each descriptor states was assigned with a rank which was used for our morphological analysis.

Statistical analysis was carried out using INDOSTAT package developed by Indostat Services, Hyderabad, India.

\section{Multivariate analysis} performed.

Classification (cluster) and ordination (Principal Component Analysis) analysis were

\section{Cluster analysis}

Simple matching similarity index was used to form clusters based on various quantitative and qualitative characters traits of 12 isolates.

For phylogenetic linkage study Weighted Average Linkage Clustering was done. This method takes weighted average of resemblance coefficients when revising the resemblance matrix. For example, suppose we are using a resemblance coefficient with values denoted by $\mathrm{R}_{\mathrm{jk}}$, under weighted average linkage clustering method, the value of coefficient between say, clusters 124 (i.e. containing accessions 1,2,4) and 35 (i.e. containing accessions 3,5) is

$\mathrm{R}_{\text {(124) (35) }}=\mathrm{w}_{13} \mathrm{R}_{13}+\mathrm{w}_{15} \mathrm{R}_{15}+\mathrm{w}_{23} \mathrm{R}_{23}+\mathrm{w}_{25} \mathrm{R}_{25}+\mathrm{w}_{34} \mathrm{R}_{34}+\mathrm{w}_{45} \mathrm{R}_{35}$, where the weights, $\mathrm{w}_{j k}$ are unequal.

The method gives large weights to those isolates admitted to their clusters at a more recent clustering step. This means that the earlier an isolate enters a cluster in the sequence of clustering steps, the less weight its resemblance with isolates outside its cluster is given when the resemblance between clusters is evaluated. The scored data was finally converted into binary form based on presence (1) or absence (0) for that particular character and subjected to statistical analysis using INDOSTAT package developed by Indostat services, Hyderabad, India.

\section{Dual culture test}

Invitro confrontations were studied by dual culture technique [7]. This technique was used to test the antagonistic ability of Trichoderma isolates against the phytopathogenic fungi Pythium aphanidermatum and Sclerotinia sclerotiorum. The host fungus and Trichoderma were grown on potato dextrose agar (PDA) for a week at room temperature $\left(28 \pm 2^{\circ} \mathrm{C}\right)$. Small bocks of the target fungus were cut from the periphery and transferred to the fresh petridish containing PDA. The Petridish consisting of target fungi and Trichoderma was incubated at room temperature and observed periodically.

Growth parameters in all dual cultures were read after 7 days. The assay was repeated twice. As all the isolates were having biocontrol potentiality every isolates were taken for further studies. The plates containing only the target pathogenic organisms without Trichoderma were taken as control to evaluate the percent growth inhibition. 


\section{Results}

\section{Morphological characterization}

The basic for taxonomical studies is morphological characterization which even though is a long-established technique still today it has its importance. The isolates were grown on PDA for 3 7 days as pure cultures and the various mycelial and conidial characters of different isolates were recorded as camera lucida drawings. The morphological characters were based on the fifteen characters included here viz. colony Growth rate (after 7 days in $\mathrm{cm}$ ) at $25^{\circ} \mathrm{C}$, colony colour, reverse colony colour, colony edge, culture smell, conidiation, mycelial form, mycelial colour, conidiophore branching, phialide disposition, phialide shape, conidial shape, conidial wall etc (Table 2). The morphological observations of the twelve isolates can be recapitulated as follows. Most of the isolates belonging to T.harzianum were similar in colony colour, culture smell, mycelial colour, conidiation, conidial shape, conidial wall and conidial colour (Plate 1). Similarly the isolates of T.viride showed certain similarity in colony colour, colony edge, culture smell, conidiophore branching, conidial wall, conidial colour and chlamydospores (Plate 2). The major difference between the isolates of T.harzianum and T.viride were their conidial wall pattern, conidial shape, conidial colour, colony edge and culture smell.

Table 2. Descriptions and morphological characters of the specios used for statistical analysis

\begin{tabular}{|c|c|c|c|c|c|c|c|c|c|c|c|c|c|}
\hline & $\begin{array}{c}\text { Isolated } \\
\text { characters }\end{array}$ & Th3 & Th10 & Th30 & Th31 & ThAg & Tv2 & Tv4 & Tr12 & Tv15 & Tv32 & TrChen & TvNir \\
\hline \multirow{5}{*}{ 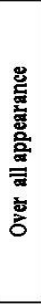 } & $\begin{array}{l}\text { Colony } \\
\text { growth } \\
\text { rate (cm) }\end{array}$ & 8-9 in 3days & $\begin{array}{l}8-9 \text { in } \\
\text { 3days }\end{array}$ & $\begin{array}{l}\text { 8-9 in } \\
\text { 3days }\end{array}$ & $\begin{array}{l}\text { 8-9 in } \\
\text { 3days }\end{array}$ & $\begin{array}{l}\text { 9-10cmin } \\
\text { 5days }\end{array}$ & $\begin{array}{l}9-10 \mathrm{cmin} \\
5 \text { days }\end{array}$ & $\begin{array}{l}\text { 7-8cm in } \\
\text { 5days }\end{array}$ & $\begin{array}{l}6-7 \mathrm{~cm} \text { in } \\
5 \text { days }\end{array}$ & $\begin{array}{l}9-10 \mathrm{cmin} \\
5 \text { days }\end{array}$ & $\begin{array}{l}\text { 9-10cmin } \\
\text { 5days }\end{array}$ & $\begin{array}{l}\text { 9-10cmin } \\
\text { 5days }\end{array}$ & $\begin{array}{l}\text { 9-10cmin } \\
\text { Sdays }\end{array}$ \\
\hline & \begin{tabular}{|l} 
Colony \\
colour
\end{tabular} & \begin{tabular}{|l|} 
Green to \\
dark green
\end{tabular} & \begin{tabular}{|l}
$\begin{array}{l}\text { Green to } \\
\text { dark green }\end{array}$ \\
\end{tabular} & $\begin{array}{l}\text { Green to } \\
\text { dark green }\end{array}$ & \begin{tabular}{|l}
$\begin{array}{l}\text { Green to } \\
\text { dark green }\end{array}$ \\
\end{tabular} & Dark green & Dark green & \begin{tabular}{|l|} 
Dark green \\
\end{tabular} & \begin{tabular}{|l} 
Yellow to \\
green
\end{tabular} & \begin{tabular}{|l|} 
Olive \\
green to \\
Dark green
\end{tabular} & $\begin{array}{l}\text { Yellow to } \\
\text { green }\end{array}$ & \begin{tabular}{|l|} 
Yellow to \\
green
\end{tabular} & Dark green \\
\hline & $\begin{array}{l}\text { Reverse } \\
\text { colony } \\
\text { colour }\end{array}$ & Yellow & \begin{tabular}{|l} 
Colourless \\
\end{tabular} & $\begin{array}{l}\text { Light } \\
\text { ycllow }\end{array}$ & Creamish & \begin{tabular}{|l} 
Deep \\
ycllow
\end{tabular} & \begin{tabular}{|l} 
Deep \\
ycllow
\end{tabular} & \begin{tabular}{|l|} 
Colourless \\
\end{tabular} & \begin{tabular}{|l|} 
Deep \\
yellow
\end{tabular} & Colourless & \begin{tabular}{|l|} 
Deep \\
yellow
\end{tabular} & \begin{tabular}{|l|} 
Colourless \\
\end{tabular} & \begin{tabular}{|l|} 
Deep \\
yellow
\end{tabular} \\
\hline & \begin{tabular}{|l|} 
Colony \\
edge
\end{tabular} & Smooth & Smooth & Smooth & Smooth & Wavy & Wavy & Wavy & Wavy & Wavy & Smooth & Wavy & Wavy \\
\hline & \begin{tabular}{|l|}
$\begin{array}{l}\text { Culture } \\
\text { smell }\end{array}$ \\
\end{tabular} & Malt & Malt & Malt & Malt & Coc & Cooc & Coc & Cock & Coconut & Coconut & Coconut & Coconut \\
\hline \multirow{2}{*}{ 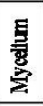 } & \begin{tabular}{|l} 
Mycelial \\
form
\end{tabular} & Arachnoid & $\begin{array}{l}\text { Floccoseto } \\
\text { Arachnoid }\end{array}$ & $\begin{array}{l}\text { Floccose to } \\
\text { Arachnoid }\end{array}$ & $\begin{array}{l}\text { Floccoseto } \\
\text { Arachnoid }\end{array}$ & $\begin{array}{l}\text { Floccose to } \\
\text { Arachnoid }\end{array}$ & Arachnoid & Arachnoid & Floccose & Floccose & Floccose & Floccose & $\begin{array}{l}\text { Floccoset to } \\
\text { Arachnoid }\end{array}$ \\
\hline & \begin{tabular}{|l} 
Mycelial \\
colour
\end{tabular} & $\begin{array}{l}\text { Watery } \\
\text { white }\end{array}$ & \begin{tabular}{|l|} 
Watery \\
white
\end{tabular} & $\begin{array}{l}\text { Watery } \\
\text { white }\end{array}$ & $\begin{array}{l}\text { Watery } \\
\text { white }\end{array}$ & $\begin{array}{l}\text { Watery } \\
\text { white }\end{array}$ & $\begin{array}{l}\text { Watery } \\
\text { white }\end{array}$ & White & White & \begin{tabular}{|l|} 
Watery \\
white
\end{tabular} & $\begin{array}{l}\text { Natery } \\
\text { vhite }\end{array}$ & White & $\begin{array}{l}\text { Watery } \\
\text { white }\end{array}$ \\
\hline \multirow[b]{2}{*}{$\begin{array}{l}\text { 홓 } \\
\text { 흘 } \\
\text { 홀 }\end{array}$} & \begin{tabular}{|l|l} 
Conidiation \\
\end{tabular} & \begin{tabular}{|l|}
$\begin{array}{l}\text { Ring like } \\
\text { zones }\end{array}$ \\
\end{tabular} & \begin{tabular}{|l|} 
Ring like \\
zones
\end{tabular} & \begin{tabular}{|l|}
$\begin{array}{l}\text { Ring like } \\
\text { zones }\end{array}$ \\
\end{tabular} & \begin{tabular}{|l|}
$\begin{array}{l}\text { Ring like } \\
\text { zones }\end{array}$ \\
\end{tabular} & $\begin{array}{l}\text { Concentric } \\
\text { zones }\end{array}$ & $\begin{array}{l}\text { Concentric } \\
\text { zones }\end{array}$ & \begin{tabular}{|l|}
$\begin{array}{l}\text { Concentric } \\
\text { zones }\end{array}$ \\
\end{tabular} & \begin{tabular}{|l|}
$\begin{array}{l}\text { Ring like } \\
\text { zones }\end{array}$ \\
\end{tabular} & \begin{tabular}{|l|}
$\begin{array}{l}\text { Ringlike } \\
\text { zones }\end{array}$ \\
\end{tabular} & \begin{tabular}{|l|} 
Ring like \\
zones
\end{tabular} & \begin{tabular}{|l|}
$\begin{array}{l}\text { Concentric } \\
\text { zones }\end{array}$ \\
\end{tabular} & $\begin{array}{l}\text { Concentric } \\
\text { zones }\end{array}$ \\
\hline & $\begin{array}{l}\text { Conidiopho } \\
\text { re } \\
\text { branching }\end{array}$ & \begin{tabular}{|l|} 
Highly \\
branched, \\
regular \\
2-3 $\mu \mathrm{mL}$
\end{tabular} & $\begin{array}{l}\text { Highly } \\
\text { branched, } \\
\text { regular } \\
4-6 \mu \mathrm{mL}\end{array}$ & $\begin{array}{l}\text { Branched, } \\
\text { regular } \\
\text { 1-2 } \mathrm{mLL}\end{array}$ & $\begin{array}{l}\text { Branched, } \\
\text { regular } \\
1-3 \mu \mathrm{mL}\end{array}$ & \begin{tabular}{|l|} 
Moderately \\
branched, \\
irregular \\
$4-5 \mu \mathrm{mL}$
\end{tabular} & \begin{tabular}{|l|} 
Moderately \\
branched, \\
imregular \\
$2-2.5 \mu \mathrm{mL}$
\end{tabular} & \begin{tabular}{|l|} 
Moderately \\
branched, \\
irregular \\
4-5pmL
\end{tabular} & \begin{tabular}{|l|} 
Moderately \\
branched, \\
irregular \\
$4-5 \mu \mathrm{mL}$
\end{tabular} & \begin{tabular}{|l|} 
Moderately \\
branched, \\
irregular \\
$1.5-2.5 \mu \mathrm{m}$ \\
$\mathrm{L}$ \\
\end{tabular} & \begin{tabular}{|l|} 
Highly \\
branched, \\
irregular \\
$1.5-2.5 \mu \mathrm{m}$ \\
L \\
\end{tabular} & \begin{tabular}{|l|} 
Hïghly \\
branched, \\
irregular \\
$1.5-2.5 \mu \mathrm{m}$ \\
$\mathrm{L}$ \\
\end{tabular} & \begin{tabular}{|l} 
Moderately \\
branched, \\
irregular \\
$4-5 \mu \mathrm{mL}$
\end{tabular} \\
\hline \multirow[b]{2}{*}{$\begin{array}{l}\text { 플 } \\
\text { 롬 }\end{array}$} & \begin{tabular}{|l|} 
Phialisle \\
disposition
\end{tabular} & 2-3 whorls & Solitary & $2 / 3$ whorls & 2-3 whorls & Solitary & $2 / 3$ whorls & $2 / 3$ whorls & $2 / 3$ whorls & Solitary & Solitary & Solitary & Solitary \\
\hline & $\begin{array}{l}\text { Phialide } \\
\text { shape }\end{array}$ & \begin{tabular}{|l|} 
Globose \\
$8-15 \times 2-3$ \\
$\mu \mathrm{m}$
\end{tabular} & $\begin{array}{l}\text { Nine-Pin } \\
\text { shape } \\
8-15 \times 2-3 \\
\text { Lm }\end{array}$ & $\begin{array}{l}\text { Nine-Pin } \\
\text { shape } \\
8-14 x \\
2.4-3 \text { mm }\end{array}$ & \begin{tabular}{|l|} 
Globose \\
$8-15 \times 2-3$ \\
$\mu \mathrm{m}$
\end{tabular} & \begin{tabular}{|l|} 
Sigmoid or \\
hooked \\
$8-14 \mathrm{x}$ \\
$2.4-3 \mu \mathrm{m}$ \\
\end{tabular} & \begin{tabular}{|l|}
$\begin{array}{l}\text { Sigmoid or } \\
\text { hooked }\end{array}$ \\
$\mathbf{8}-14 \mathrm{x}$ \\
$\mathbf{2 - 3 . 5} \mathrm{\mu m}$ \\
\end{tabular} & \begin{tabular}{|l|} 
Sigmoid or \\
hooked \\
$8-14 \mathrm{x}$ \\
$2-3 \mathrm{\mu m}$ \\
\end{tabular} & \begin{tabular}{|l|} 
Sigmoid or \\
hooked \\
$8-14 \mathrm{x}$ \\
$2.4-3 \mathrm{\mu m}$ \\
\end{tabular} & 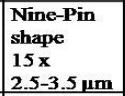 & \begin{tabular}{|l|} 
Nine-Pin \\
shape \\
$4.5-12.5 \mathrm{x}$ \\
$2.5-3.5 \mathrm{~mm}$
\end{tabular} & $\begin{array}{l}\text { Nine-Pin } \\
\text { shape } \\
5-18 x \\
2-3.5 \mu \mathrm{m}\end{array}$ & \begin{tabular}{|l|} 
Sigmoid or \\
hooked \\
$8-14 \mathrm{x}$ \\
$2.4-3 \mu \mathrm{m}$ \\
\end{tabular} \\
\hline \multirow{3}{*}{ 홀 } & \begin{tabular}{|l} 
Conidial \\
shape
\end{tabular} & \begin{tabular}{|l|} 
Subglobose \\
$3.6-4.5 \mu \mathrm{m}$
\end{tabular} & $\begin{array}{l}\text { Subglobos } \\
\text { e } \\
3.6-1.5 \mu \mathrm{m}\end{array}$ & $\begin{array}{l}\text { Subglobos } \\
\text { e } \\
3-4.8 \times 3.5- \\
4 \mathrm{\mu m} \\
\end{array}$ & $\begin{array}{l}\text { Subglobos } \\
\text { e } \\
3.5-4.5 \mu \mathrm{m}\end{array}$ & $\begin{array}{l}\begin{array}{l}\text { Globose to } \\
\text { obovoid } \\
3.6-4.5 \mu \mathrm{m}\end{array} \\
\end{array}$ & $\begin{array}{l}\text { Globose to } \\
\text { obovoid } \\
3-4.5 \mathrm{~mm}\end{array}$ & $\begin{array}{l}\begin{array}{l}\text { Globose to } \\
\text { obovoid } \\
3.6-4.5 \\
\mathrm{~mm}\end{array} \\
\end{array}$ & $\begin{array}{l}\text { Globose to } \\
\text { subglobose } \\
3.6-4.5 \mathrm{\mu m}\end{array}$ & $\begin{array}{l}\text { Globose to } \\
\text { subglobose } \\
3-5 \mathrm{~mm}\end{array}$ & $\begin{array}{l}\text { Globose to } \\
\text { subglobose } \\
2.5-3.5 \mu \mathrm{m}\end{array}$ & $\begin{array}{l}\text { Globose to } \\
\text { subglobose } \\
2.5-5 \mu \mathrm{m}\end{array}$ & $\begin{array}{l}\begin{array}{l}\text { Globose to } \\
\text { obovoid } \\
3.6-4.5 \mu \mathrm{m}\end{array} \\
\end{array}$ \\
\hline & \begin{tabular}{|l|} 
Conidial \\
wall
\end{tabular} & Rough & Rough & Rough & Rough & \begin{tabular}{|l|} 
Smooth \\
\end{tabular} & \begin{tabular}{|l|} 
Smooth \\
\end{tabular} & Smooth & \begin{tabular}{|l|l|} 
Smooth \\
\end{tabular} & Smooth & Smooth & Smooth & Smooth \\
\hline & \begin{tabular}{|l|} 
Conidial \\
colour
\end{tabular} & Green & Green & Green & Green & Green & Green & Green & Green & $\begin{array}{l}\text { Dark } \\
\text { Green }\end{array}$ & \begin{tabular}{|l|} 
Dark \\
Green
\end{tabular} & \begin{tabular}{|l|} 
Dark \\
Green \\
\end{tabular} & Green \\
\hline \multicolumn{2}{|c|}{ Chlamydospores } & \begin{tabular}{|l|} 
Not \\
observed
\end{tabular} & \begin{tabular}{|l|}
$\begin{array}{l}\text { Not } \\
\text { observed }\end{array}$ \\
\end{tabular} & $\begin{array}{l}\text { Present } \\
\text { globose }\end{array}$ & \begin{tabular}{|l|} 
Not \\
observed
\end{tabular} & $\begin{array}{l}\text { Not } \\
\text { observed }\end{array}$ & $\begin{array}{l}\text { Not } \\
\text { observed }\end{array}$ & \begin{tabular}{|l|} 
Not \\
observed
\end{tabular} & \begin{tabular}{|l|} 
Not \\
observed
\end{tabular} & \begin{tabular}{|l|} 
Not \\
observed
\end{tabular} & $\begin{array}{l}\text { Not } \\
\text { observed }\end{array}$ & \begin{tabular}{|l|}
$\begin{array}{l}\text { Not } \\
\text { observed }\end{array}$ \\
\end{tabular} & \begin{tabular}{|l|} 
Not \\
observed
\end{tabular} \\
\hline
\end{tabular}




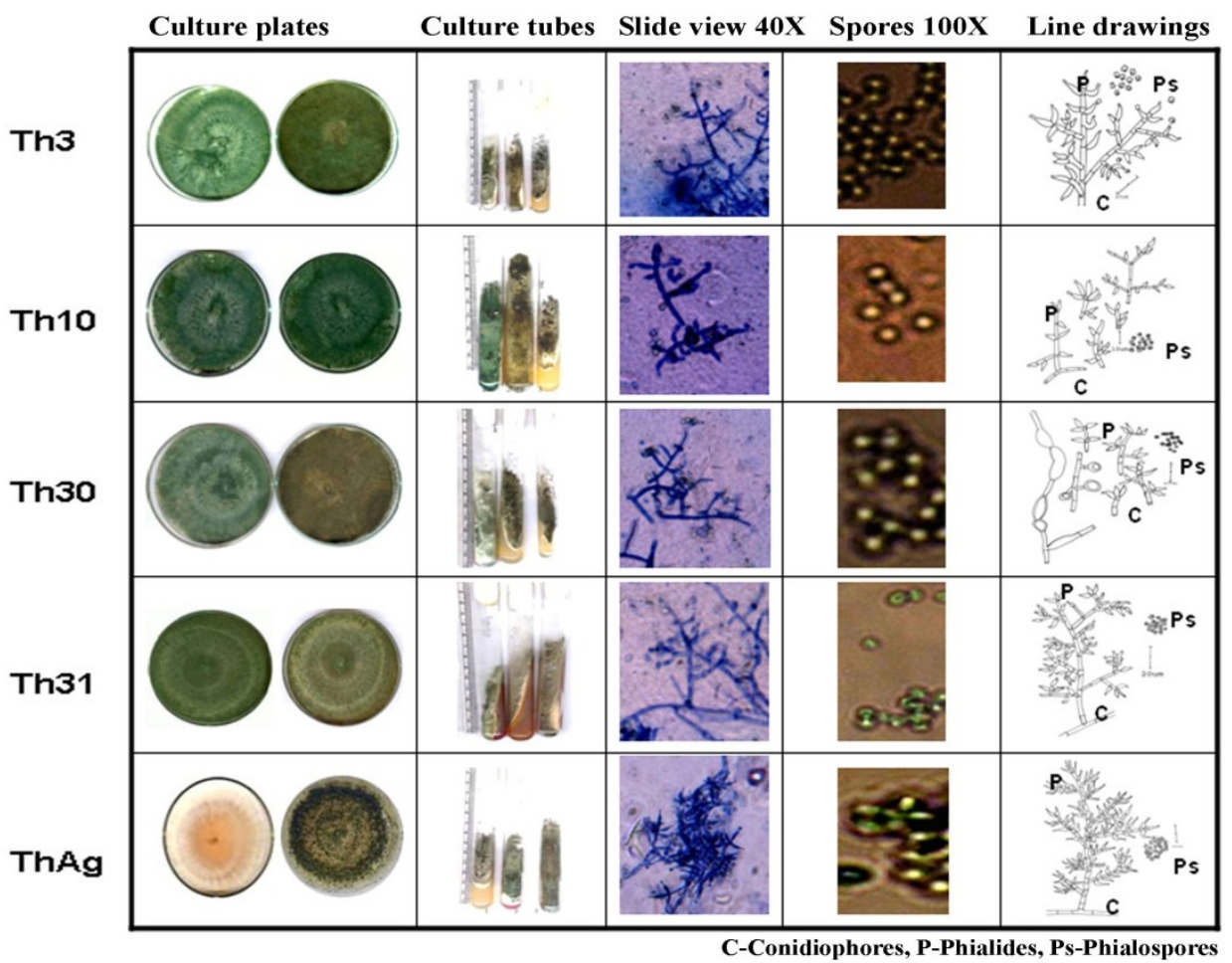

Plate 1. Morphological observation of T.harzianum isolates (slide view - light microscope, scale for camera lucida drawings $20 \mu \mathrm{m}$ )

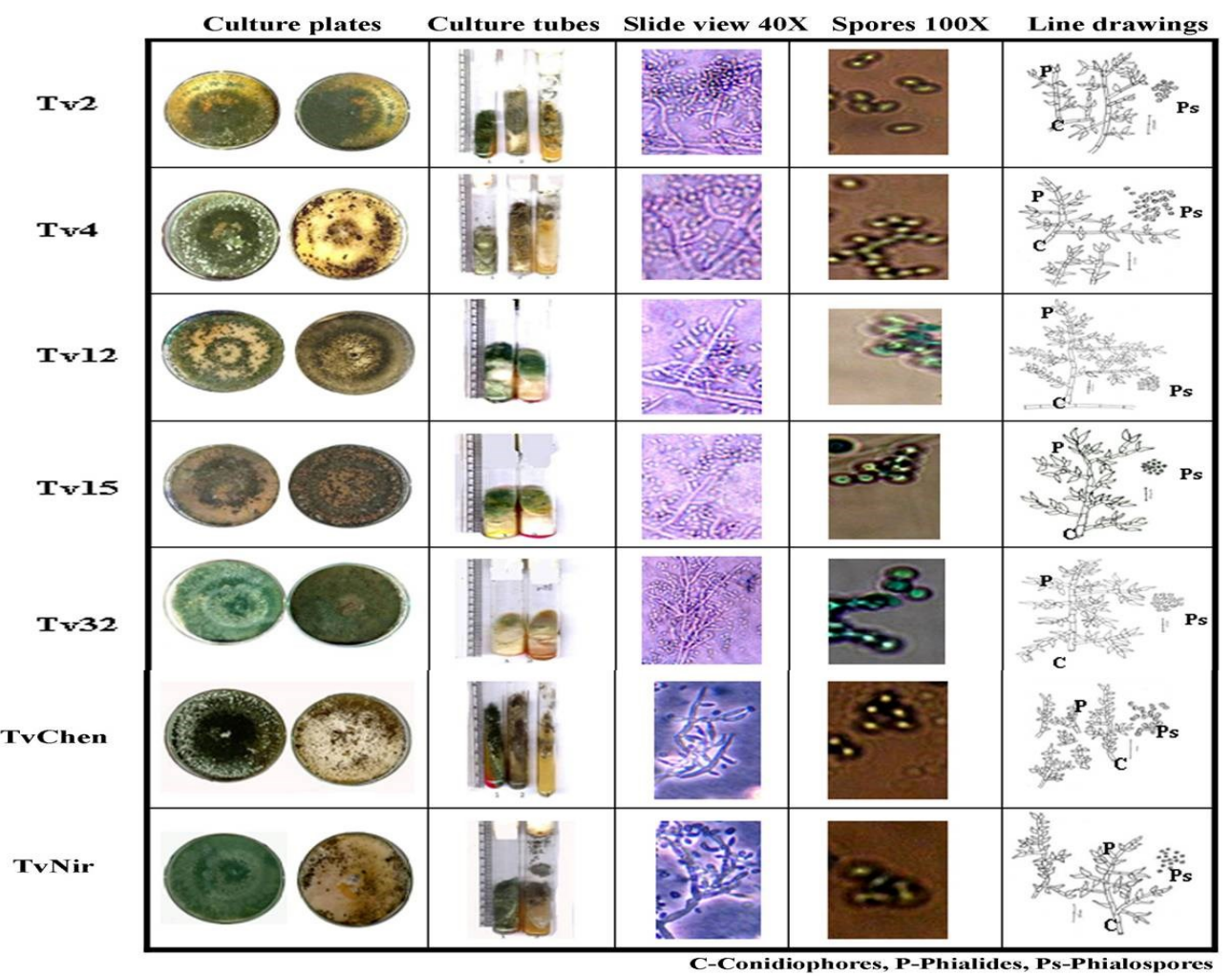

Plate 2. Morphological observation of T.viride isolates (slide view - light microscope, scale for camera lucida drawings $20 \mu \mathrm{m}$ ) 


\section{Cluster analysis}

As mentioned above, only few characters were significantly differing between the two species, a total of fifteen quantitative and qualitative characters were taken to analyze the inter specific difference. However most of characters observed were in abstract nature which may hinder the statistical analysis of the isolates. Thus variation in the characters was given specific ranking so as to facilitate the comparative study. These numerical data was again converted to binary form, which was subjected to statistical analysis. This was based on the report by Samuel et al. where he used the morphological characterization to analyze the variation between the isolates among Trichoderma species associated with green mold epidemic of commercially grown Agaricus bisporus [15]. Similarly Munaut et al. reported variation in Colletotrichum gloeosporioides based on morphological characterization [12]. The data were used to compute simple matching similarity index and dendrogram was constructed which is shown in Fig. 1. Three distinct clusters were obtained and the morphological similarity among the isolates varied from $40-90 \%$. The salient features of the clusters are summarized below.

Cluster 1 This cluster consists of all the isolates of T.harzianum characterized by colony colour, culture smell, mycelial colour, conidiation, conidial shape, conidial wall and conidial colour.

Cluster 2 Four isolates of T.viride formed this cluster having characters like similar colony edge, culture smell, conidiophore branching, phialide shape, conidial wall and chlamydospores.

Cluster 3 Tv32, TvChen and TvNir belonging to T.viride formed this cluster which consisted of similar growth rate, colony colour, mycelial form, phialide disposition, phialide shape, conidial shape, conidial wall, conidial colour and chlamydospores.

The cluster analysis grouped the twelve isolates into three major clusters. The inter and intra cluster distances are shown in Table 3. The inter cluster distance between clusters 1 and 3 was the highest $(0.605)$ followed by cluster 1 and $2(0.458)$. Intracluster distance was highest for cluster 2 (0.332) followed by cluster $1(0.20)$ and cluster $3(0.175)$.From the intra cluster distances it can be inferred that the cluster 2 was more diverse than cluster 1 and 3 which was supported by shaded matrix index. The inter cluster distances showed that cluster 1 was highly distant from cluster 3 followed by cluster 2. This finding confirmed the result of cluster analysis where T.harzianum formed an entirely distinct group from the isolates of T.viride. The result of PCA was also in consistent with this finding.

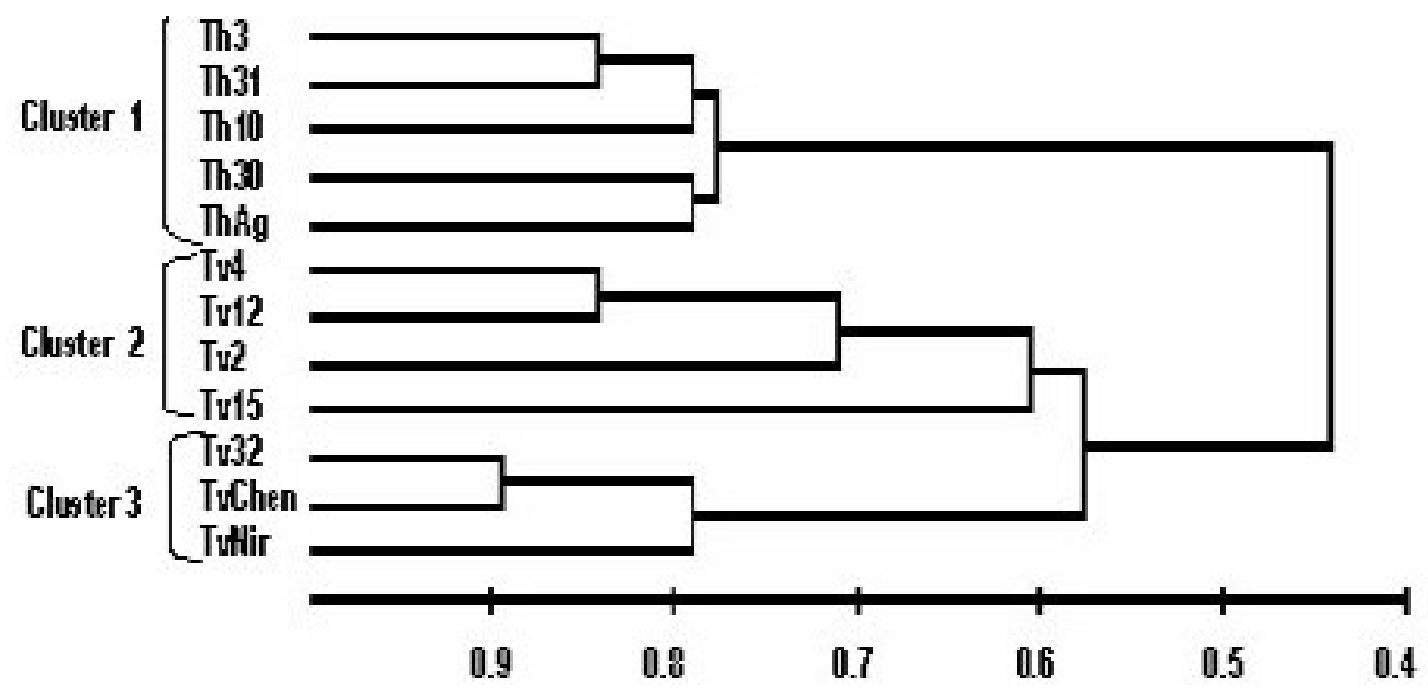

Fig 1. Inter-relationship between the Trichoderma isolates based on the morphological characters. The value $0.4-0.9$ indicates the simple matrix similarity index. 
Table 3. Inter and Intra cluster distances for the isolates of Trichoderma isolates.

\begin{tabular}{|c|c|c|c|}
\hline Clusters & Cluster 1 & Cluster 2 & Cluster 3 \\
\hline Cluster 1 & 0.202 & $\mathbf{0 . 4 5 8}$ & $\mathbf{0 . 6 0 5}$ \\
\hline Cluster 2 & & 0.332 & $\mathbf{0 . 4 5 3}$ \\
\hline Cluster 3 & & & 0.175 \\
\hline
\end{tabular}

\section{Similarity matrix}

A band graph analysis was carried out for the 12 isolates. The graphic depiction showed similar band alignment for the isolates with similar characters (Fig. 2).

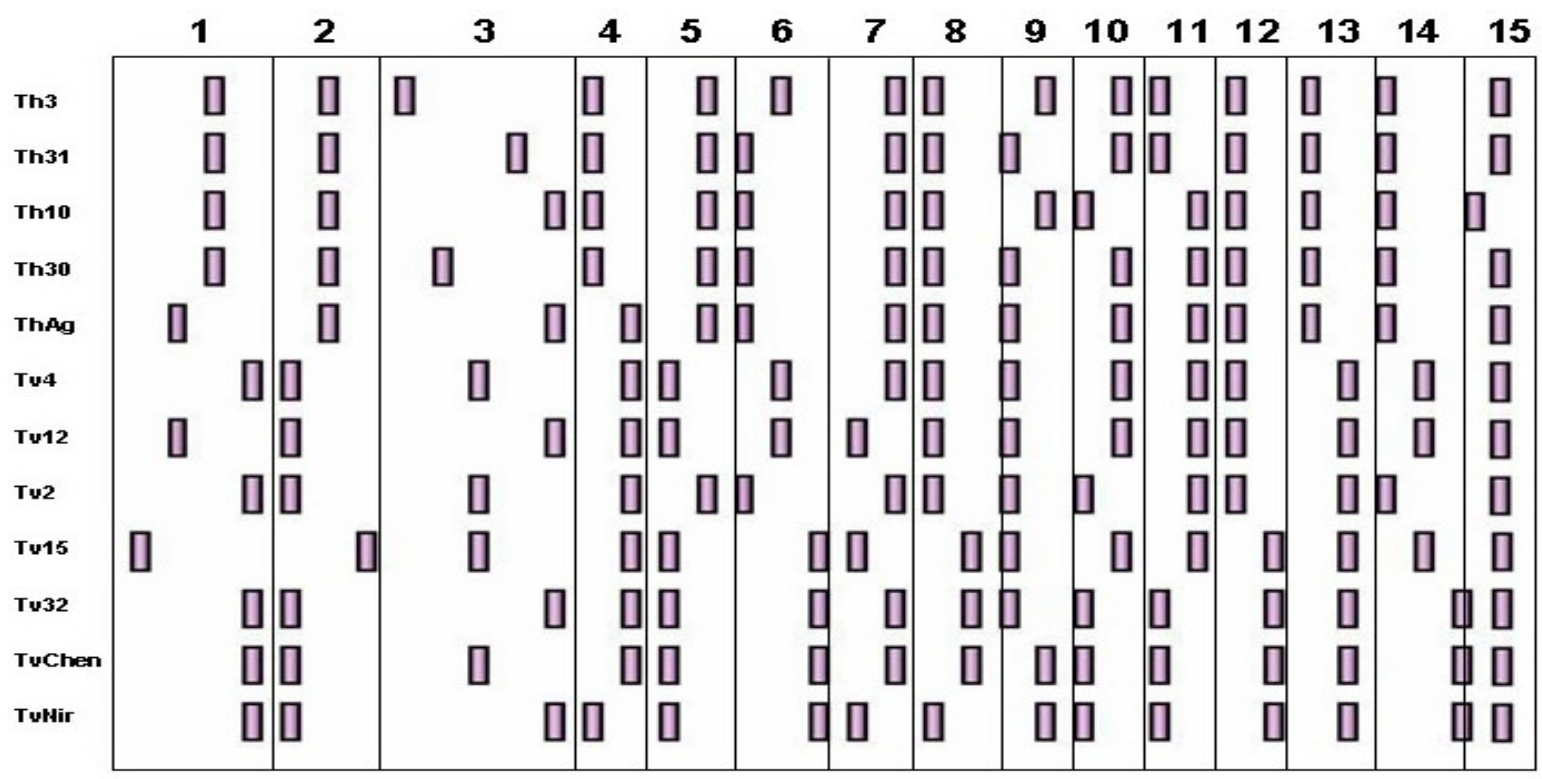

Fig 2. Graphical depiction of the descriptors for the 12 Trichoderma isolates. No's 1-15 are the fifteen quantitative and qualitative characters taken for statistical analysis

The shaded similarity matrix was used to make pair wise comparison for all isolates to confirm their similarity. The dark colour region of the matrix showed the maximum similarity of $90-100 \%$ between the isolates while the light colored region showed the minimum similarity between the isolates (Fig. 3). The maximum similarity was between the isolates Tv12 and Tv4, while minimum similarity was between isolates TvChen and Th30. 


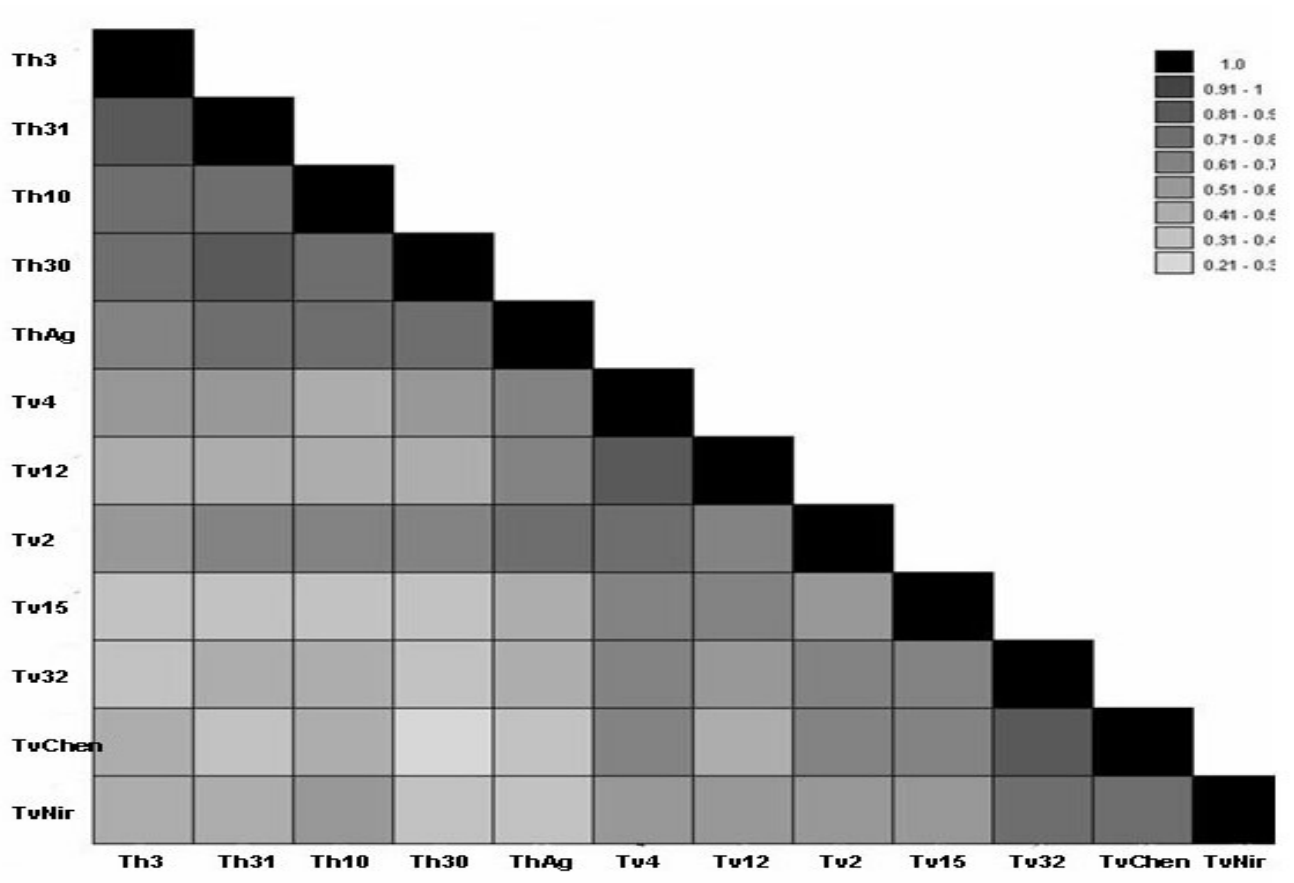

Fig 3. Graphical representation of the pair wise similarity index between the Trichoderma isolates

\section{Principle component analysis (PCA)}

PCA performed on studied traits showed that first two most informative components accounted for about $58 \%$ variation and the plot showed T.harzianum and T.viride as two distinct components confirming the cluster analysis (Fig. 4).

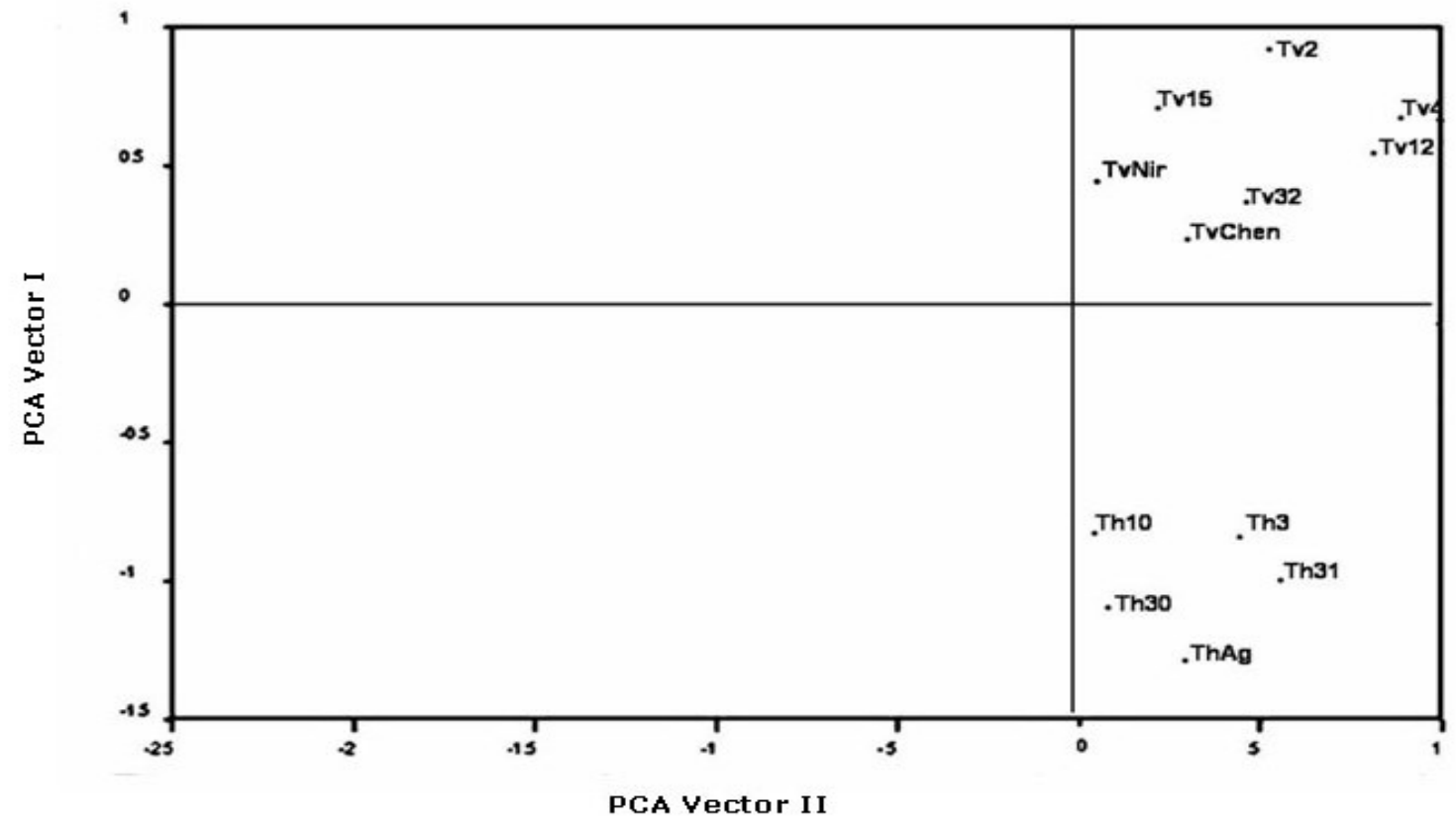

Fig 4. Principal Component Analysis of the Trichoderma isolates based on morphology. The vectors I and II are the first two most informative components accounted for about 58\% variation 


\section{Biocontrol efficacy}

The Bioefficacy of Trichoderma has been reviewed by several authors, and reported the biocontrol potential over different plant pathogens [21, 10, and 8]. As we know that Trichoderma is one of the organisms that grow well under laboratory conditions; it was easy to perform the confrontation assay through dual culture with the soil borne pathogens Pythium aphanidermatum and Sclerotinia sclerotiorum. The isolates taken for the present study has been already established as biocontrol agents by various antagonistic and biochemical studies [16]. The Bioefficacy of these isolates were again confirmed by dual culture assay.

The confrontation assay to study the antagonistic effect of Trichoderma isolates on Pythium aphanidermatum and Sclerotinia sclerotiorum was done. The study revealed that T.harzianum isolates were more aggressive in checking the growth of the pathogenic fungi than T.viride. Among the T.harzianum isolates, Th3 had more profuse growth of $23 \mathrm{~cm}^{2}$ which overlapped P.aphanidermatum inhibiting its growth by $86 \%$ which was followed by Th10 and Th31. The isolate ThAg had the least effect of $74 \%$ among the T.harzianum isolates. TvChen had highest antagonistic property among the T.viride isolates against P.aphanidermatum which was followed by Tv4 (75\%) and TvNir (74\%). Similar results were obtained against S.sclerotiorum also, where Th3 exhibited $90 \%$ inhibition followed by Th10 and Th30. The isolate TvNir had maximum impact of $85 \%$ against the target fungi, followed by Tv2, TvChen and Tv4 (Table 4). The biocontrol potentiality of the Trichoderma isolates obtained in the confrontation assay was in consistent with the earlier reports of Sharma et al.,Sharma and Sain, Sharma and Dureja [20,18, 19, 17]. Now an attempt was made to corroborate phenetic characters with the antagonistic ability.

Table 4. Bioefficacy of Trichoderma isolates on Pythium aphanidermatum and Sclerotinia sclerotiorum.

\begin{tabular}{|c|c|c|c|c|c|}
\hline \multirow[b]{2}{*}{ Isolate no: } & \multirow[b]{2}{*}{ Trichoderma sp. } & \multicolumn{2}{|c|}{ Pythium aphanidermatum } & \multicolumn{2}{|c|}{ Sclerotinia sclerotiorum } \\
\hline & & $\begin{array}{c}\text { Percent } \\
\text { inhibition of } \\
\text { mycelial } \\
\text { growth }(\%) \\
\text { of pathogen }\end{array}$ & $\begin{array}{l}\text { Mean mycelial } \\
\text { growth }\left(\mathrm{cm}^{2}\right) \text { of } \\
\text { Trichoderma }\end{array}$ & $\begin{array}{c}\text { Percent } \\
\text { inhibition of } \\
\text { mycelial } \\
\text { growth (\%) } \\
\text { of pathogen }\end{array}$ & $\begin{array}{l}\text { Mean mycelial } \\
\text { growth }\left(\mathrm{cm}^{2}\right) \text { of } \\
\text { Trichoderma }\end{array}$ \\
\hline Th3 & $\begin{array}{c}\text { Trichoderma } \\
\text { harzianum }\end{array}$ & 86.4 & 23.04 & 90.2 & 25.00 \\
\hline Th10 & $\begin{array}{c}\text { Trichoderma } \\
\text { harzianum }\end{array}$ & 83.0 & 21.16 & 88.1 & 24.01 \\
\hline Th30 & $\begin{array}{c}\text { Trichoderma } \\
\text { harzianum }\end{array}$ & 78.3 & 18.49 & 85.0 & 23.04 \\
\hline Th31 & $\begin{array}{c}\text { Trichoderma } \\
\text { harzianum }\end{array}$ & 80.0 & 20.25 & 74.5 & 16.81 \\
\hline ThAg & $\begin{array}{c}\text { Trichoderma } \\
\text { harzianum }\end{array}$ & 74.5 & 16.81 & 72.7 & 14.40 \\
\hline Tv2 & Trichoderma viride & 68.0 & 14.44 & 83.3 & 23.24 \\
\hline Tv4 & Trichoderma viride & 75.0 & 16.81 & 80.5 & 18.49 \\
\hline Tv12 & Trichoderma viride & 70.0 & 15.21 & 74.7 & 16.81 \\
\hline Tv15 & Trichoderma viride & 56.5 & 12.96 & 60.9 & 12.96 \\
\hline Tv32 & Trichoderma viride & 65.8 & 14.44 & 50.5 & 12.25 \\
\hline TvChen & Trichoderma viride & 77.0 & 17.64 & 82.0 & 20.25 \\
\hline
\end{tabular}




\section{Discussion}

Thus the morphological observations of the twelve isolates can be recapitulated as follows. Most of the isolates belonging to T.harzianum were similar in colony colour, culture smell, mycelial colour, conidiation, conidial shape, conidial wall and conidial colour. Similarly the isolates of T.viride showed certain similarity in colony colour, colony edge, culture smell, conidiophore branching, conidial wall, conidial colour and chlamydospores. The major difference between the isolates of T.harzianum and T.viride were their conidial wall pattern, conidial shape, conidial colour, colony edge and culture smell.

As mentioned above, only few characters were significantly differing between the two species, a total of fifteen quantitative and qualitative characters were taken to analyze the inter specific difference. However most of characters observed were in abstract nature which may hinder the statistical analysis of the isolates. Thus variation in the characters was given specific ranking so as to facilitate the comparative study. These numerical data was again converted to binary form, which was subjected to statistical analysis. This was based on the report by Samuel et al. where he used the morphological characterization to analyze the variation between the isolates among Trichoderma species associated with green mold epidemic of commercially grown Agaricus bisporus [15]. Similarly Munaut et al. reported variation in Colletotrichum gloeosporioides based on morphological characterization [12].

The cluster analysis illustrated all the isolates of T.harzianum formed a single cluster while the isolates of T.viride were bifurcated into two groups. The main contributing characters which distinguished the T.viride isolates were phialide shape, conidial shape and conidial colour. The clustering was substantiated by similarity index which showed maximum similarity among T.harzianum isolates with only less than $20 \%$ variation among themselves. Similarly the clusters having isolates of T.viride also had less variation within them. However, when the cluster 1 constituting all T.harzianum isolates compared with cluster 2 consisting of Tv4, Tv12, Tv2 and Tv15, they shared only 50\% similarity and when the cluster 1 was compared with cluster 3 having Tv32, TvChen and TvNir they had only $20 \%$ in common.

From the intra cluster distances it can be inferred that the cluster 2 was more diverse than cluster 1 and 3 which was supported by shaded matrix index. The inter cluster distances showed that cluster 1 was highly distant from cluster 3 followed by cluster 2 . This finding confirmed the result of cluster analysis where T.harzianum formed an entirely distinct group from the isolates of T.viride. The result of PCA was also in consistent with this finding.

\section{Relation between morphology and biocontrol efficacy}

The morphological markers grouped all T.harzianum isolates together and did not establish much variation within these isolates. Nevertheless these isolates varied in their pathogenic ability against the two target fungi studied. The isolate Th3 which was found to be best biocontrol agent through confrontation study, was unable to differentiate itself from other isolates with morphological markers. Thus no relation could be established between biocontrol efficacy and phenetic characters. On the other hand the isolates TvChen and TvNir which had higher antagonistic effect against the target fungi formed a separate group during the cluster analysis. Therefore it can be inferred that these isolates differed from other isolates at phenetic level which can be related to their pathogenic ability. Even though the present study established a vague relationship between bioefficacy and phenetic diversity it can be confirmed only when the biochemical factors like chitinase, antibiotics etc are studied in detail.

\section{Acknowledgement}

I like to express gratitude to my chairperson Pratibha sharma and CSIR in accomplishing the research work efficiently. 


\section{References}

[1] G.R. Bisby, Trichoderma viride Pers. ex Fries, and notes on Hypocrea, Trans. Brit. Mycol. Soc. 23 (1939) 149-168.

[2] J. Bissett, A revision of the genus Trichoderma. I. Section Longibrachiatum sect, Nov. Can. J. Bot. 62 (1984) 924-931.

[3] J. Bissett, A revision of the genus Trichoderma. II. Infrageneric classification, Can. J. Bot. 69 (1991a) 2357-2372.

[4] J. Bissett, A revision of the genus Trichoderma. III. .Sect. Pachybasium, Can. J. Bot.69 (1991b) 2373-2417.

[5] J. Bissett, A revision of the genus Trichoderma. IV. Additional notes on section Longibrachiatum. Can. J. Bot. 69 (1991c) 2418-2420.

[6] J. Bissett, Trichoderma atroviride, Can. J. Bot. 70 (1992) 639-641.

[7] C. Dennis and J. Webster, Antagonistic properties of species group of Trichoderma.1. Production of non-volatile antibiotics, Trans. Brit. Mycol. Soc. 57 (1971) 25-39.

[8] E.L. Ghisalberti and K. Sivasithamparam, The role of secondary metabolites produced by Trichoderma species in biological control (abstract), Petria. 1 (1991) 130-131.

[9] L. Hjeljord and A. Tronsmo, Trichoderma and Gliocladium in Biological Control: An Overview. In: Harman, G.E., Kubicek, C.P. (Eds.), Trichoderma and Gliocladium. Vol. 2. Enzymes, Biological Control and Commercial Applications. Taylor and Francis Ltd., London. (1998) 131-151pp.

[10] J.A. Lewis and G.C. Papavizas, Integrated control of Rhizoctonia fruit rot of cucumber, Phytopathology. 70 (1980) 85-89.

[11] E. Lieckfeldt G.J. Samuels, H.I. Helgard and O. Petrini A morphological and molecular perspective of Trichoderma viride: is it one or two species, Appl. Environ. Microbial. 65 (1999) 2418-2428.

[12] F. Munaut, N. Hamaide and H. Maraite, Molecular and morphological characterization of Colletotrichum gloeosporioides from native Mexican Stylosanthes species, Pl. Path. 50 (2001) 383-396.

[13] H.I. Nirenberg, Untersuchungen uber die morphologische und biologische differenzierung in der Fusarium Sektion Liseola, Mitt Biol Bundesanstalt fur Land-Forstw Berlin-Dallem. 169 (1976) 1-117.

[14] M.A. Rifai, A revision of the genus Trichoderma, Mycol. Papers. (1969) 116: 1-56.

[15] G.J. Samuels, S.L. Dodd, W. Gams, W, L.A. Castlebury, and O. Petrini, Trichoderma species associated with the green mold epidemic of commercially grown Agaricus bisporus. Mycologia, 94 (2002) 146-170.

[16] P. Sharma, Induction of systemic resistance to downy mildew by exogenous application of plant activator, Annals plant Prot. Sci. 10 (1) (2002) 99-103.

[17] P. Sharma, and P. Dureja, Evaluation of T.harzianum and T.viride isolates at BCA Pathogen Crop Interface, J. Mycol. Pl. Pathol. 34 (1) (2004) 47-55.

[18] P. Sharma, and S.K. Sain, Development of suitable techniques for evaluating virulence and biocontrol activity of Trichoderma isolates, Indian J. Pl. Pathol. 21 (2003) 16-21.

[19] P. Sharma, and S.K. Sain, Induction of systemic resistance in tomato and cauliflower by Trichoderma species against stalk rot pathogen. (Sclerotinia sclerotiorum), J. Biocontrol. 18 (1) (2004) 21-28.

[20] P. Sharma, S.K. Sain and S. James, Compatibility Study of Trichoderma isolates with Fungicides against Damping-off of cauliflower and tomato caused by Pythium aphanidermatum, Pesticide Research Journal. 15 (2) (2003) 133-138.

[21] A. Tronsmo and J. Ystaas, Biological control of Botrytis cinerea on apple, Plant Dis. 64 (1980) 1009.

[22] R. Weinding, Studies on a lethal principle effective in the parasitic action of Trichoderma lignorum on Rhizoctonia solani and other soil fungi, Phytopatholo. 24 (1934) 1153-1179. 